\title{
Aprendizaje colaborativo a través de la elaboración de documentales audiovisuales y el uso del edublog
}

\author{
Pere Molina; Alejandro Martínez-Baena; Joan Úbeda-Colomer; Jorge Lizandra; \\ Fernando Gómez-Gozalvo; Javier Valenciano \\ (juan.p.molina@uv.es) $)^{\mathbf{a}}$, (alejandro.martinez@uv.es) $)^{\mathbf{a}}$, (joan.ubeda-colomer@uv.es) $)^{\mathbf{a}}$,

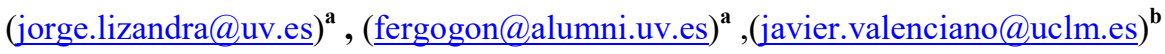 \\ ${ }^{\mathrm{a}}$ Universitat de València $\mathrm{y}^{\mathrm{b}}$ Universidad de Castilla La Mancha
}

\begin{abstract}
The aim of this work was to promote a higher implication of the students in the development of a subject from the Physical Activity and Sport Sciences bachelor belonging to University of Valencia, through a collaborative learning strategy in which the creation, in small groups, of audiovisual documentaries and their subsequent presentation in a edublog, was used.

48 students from the Ontinyent campus were involved. A high participaion of students in the experience was observed (97.96\% of the registered students), a great implication in the documentary making and also in the individual comments to them in the specific posts published in the edublog, whose average comments on these posts (25.89) was higher than the general average of comments in edublog (14.39). It was also remarkably the high quality of most of the 9 documentaries performed, considering the teacher's opinion, the content of the comments published in the post and the peer assessment of the documentaries done by the different groups.
\end{abstract}

\section{Keywords:}

Educational Innovation, ICT, participative asessment, peer assessment

\section{Resumen}

El objetivo del presente trabajo fue generar una mayor implicación del alumnado en el desarrollo de una asigantura del plan de estudios del Grado en Ciencias de la Actividad Física y el Deporte de la Universitat de València, mediante una estrategia de aprendizaje colaborativo en la que se utilizó la elaboración, en pequeños equipos, de documentales audiovisuales y su posterior comentario en un edublog. Participaron 48 estudiantes matriculados en el campus d'Ontinyent. Se constata una elevada participación del alumnado en la experiencia desarrollada (97,96\% de los matriculados), una gran implicación en la elaboración de los documentales y 
también en la realización de comentarios individuales a los mismos en posts específicos publicados en el edublog de la asignatura, cuya media de comentarios a estos posts $(25,89)$ fue mayor a la media general de comentarios a los posts del edublog (14,39). También cabe destacar la buena calidad de gran parte de los 9 documentales que se realizaron, a juzgar por la valoración del profesor de la asignatura, y también por el contenido de los comentarios publicados en los posts y por la coevaluación que realizaron los diferentes equipos sobre los documentales de sus compañeros.

Palabras clave: Innovación educativa, TIC, evaluación participativa, coevaluación

\section{Introducción}

Una de las principales innovaciones pedagógicas en Educación Superior consiste en dar protagonismo a los estudiantes, desarrollando metodologías que propicien su aprendizaje autónomo y reflexivo. Uno de los recursos para conseguirlo puede ser el uso de herramientas de la web 2.0. Por sus características (fácil manejo y participación, contenidos multimedia e interacción social), los blogs son uno de los resursos con más posibilidades didácticas. Además, permiten la ampliación del espacio físico del aula y temporal del horario de clase, resultando un instrumento adecuado para el desarrollo del trabajo no presencial y para la consecución del aprendizaje activo mediante la interacción entre el alumando.

En el curso 2009-10 un grupo de profesores de la Facultat de Ciències de la Activitat Física i l'Esport (FCAFE) de la Universitat de València (UV) decidimos constituirnos en grupo de innovación educativa. Gracias a las ayudas del Servei de Formació Permanent i Innovació Educativa (SFPIE) de dicha universidad, desde ese mismo curso y hasta la actualidad hemos ido desarrollando diversos proyectos relacionados con el uso de edublogs, cuya trayectoria hemos descrito en otros trabajos (Molina, Valencia-Peris, Valenciano, PérezSamaniego y Devís, 2012; Molina, Valenciano y Valencia-Peris, 2015). Actualmente, conformamos una red de innovación educativa relacionada con los edublogs y aprendizaje colaborativo en la que participamos 40 profesores y profesoras de diferentes departamentos de 8 universidades españolas.

En la FCAFE, concretamente, se han desarrollado experiencias en varias asignaturas en las que el uso del edublogs tenía como finalidad el servir de herramienta de ayuda al alumnado para el seguimiento del curso y de estímulo para su participación en el desarrollo de los contenidos y actividades de la asignatura (Antolín, Molina, Villamón, Devís y PérezSamaniego, 2011; Molina, Antolín, Pérez-Samaniego, Devís, Villamón y Valenciano, 2013; Molina, Valenciano y Valencia-Peris, 2015; Martínez-Baena y Molina, 2015; Molina, Valencia-Peris y Suárez, 2016). Estas experiencias obtuvieron resultados muy satisfactorios y positivos $\mathrm{y}$, actualmente, la mayoría de ellas se han integrado y forman parte de la dinámica habitual de estas asignaturas.

(cc) EY-NC-ND 2016, Universitat Politècnica de València 
La experiencia innovadora que aquí se presenta se desarrolló en la asignatura de Teoría e historia de la actividad fisica y el deporte del Grado en Ciencias de la Actividad Física y el Deporte de la UV. En el contexto español, esta asignatura es percibida como una asignatura de contenido teórico, denso y poco atractivo. Precisamente, los contenidos a los que hace referencia fueron los menos valorados por la muestra de alumnos y alumnas, en el Libro Blanco del Título de Grado en Ciencias de la Actividad Física y el Deporte (Del Villar, 2007). Por otra parte, en la experiencia docente en esta asignatura en la UV también se observaba una escasa implicación del alumnado en la elaboración de trabajos escritos, así como una falta de calidad de muchas de las aportaciones realizadas. Por estos motivos y con la finalidad de cambiar esta tendencia, en la FCAFE decidimos complementar el uso del edublog con otros recursos innovadores. Uno de ellos, y en el que nos centraremos específicamente en este trabajo, tiene que ver con la elaboración de documentales audiovisuales.

Este tipo de documentales son un instrumento metodológico que permite registrar en formato audiovisual la realidad histórica con rigor y fiabilidad, ofreciendo posibilidades estéticas y narrativas para la construcción de discursos que permitan dar a conocer los resultados obtenidos en una investigación, de una forma diferente y con un lenguaje contemporáneo (Bermúdez, 2010).

Tanto el uso del edublog de la asignatura, como la elaboración del documental audiovisual, se enmarcan dentro de un planteamiento pedagógico basado en el aprendizaje colaborativo. Este tipo de aprendizaje tiene lugar cuando un grupo de personas trabajan juntas en la resolución de problemas o tareas particulares y surge de la interacción colaborativa entre los participantes. De esta forma, cada estudiante aprende más de lo que aprendería individualmente. Se trata, por tanto, de colaborar para aprender y aprender a colaborar.

En contraste con el aprendizaje competitivo e individualista, se puede definir este tipo de aprendizaje como una forma de organizar la enseñanza por medio de la interacción de grupos reducidos cuidadosamente estructurados, en los que los alumnos trabajan juntos para maximizar su propio aprendizaje y el de los demás. Cada estudiante logra su meta sólo si los demás miembros del grupo logran las suyas. Hay una responsabilidad y un compromiso con la tarea compartida, que crea una interdependencia entre los miembros del grupo (Johnson, Jonhson y Holubec, 1998; Slavin, 1990; Kagan, 1989).

Diversos autores (Dillenbourg; 1999; Laal y Laal, 2011; Smith y McGregor, 1992; Suárez y Gros, 2013) hablan de aprendizaje colaborativo en un sentido amplio diferenciando, dentro del mismo, propuestas que van de un menor a un mayor grado de colaboración y reciprocidad. En este gradiente, el aprendizaje cooperativo es el que exige mayor colaboración y reciprocidad.

\section{Objetivos}

El principal objetivo de esta experiencia fue implicar al alumnado en el desarrollo de una asignatura que percibe como teórica, densa y poco atractiva. Para ello, se utiliza una 
estrategia de aprendizaje colaborativo consistente en la elaboración de documentales audiovisuales en equipo y la realización de comentarios sobre los mismos en un edublog de la asignatura.

\section{Desarrollo de la innovación}

La asignatura de Teoría e historia de la actividad fisica y el deporte se imparte en el primer curso del Grado en Ciencias de la Actividad Física y el Deporte de la UV. La experiencia se desarrolló, durante el segundo cuatrimestre del curso 2014-15, en el grupo del campus d'Ontinyent con 49 estudiantes matriculados.

En esta asignatura existían dos opciones de evaluación del aprendizaje del alumnado. Por una parte, una evaluación final a través de un examen sobre los contenidos de la asignatura y, por otra, una evaluación continua sumativa que atendía a los siguientes aspectos: a) $40 \%$ al examen final de la asignatura (el mismo que en la opción de evaluación final); b) 30\% a la elaboración de un documental audiovisual en equipo; y c) 30\% a la participación en el edublog a través de los comentarios del alumnado.

La experiencia que aquí se presenta tiene que ver con los aspectos b) y c), y era de especial relevancia en el desarrollo de la asignatura. Fue planteada por el profesor en los primeros días de clase como un trabajo en equipo en el que se tenía que elaborar un documental videográfico de entre $20^{\prime}$ y $25^{\prime}$ de duración. La temática de este documental era elegida por los estudiantes que podían centrarse en la biografía de un personaje clave (deportista, entrenador, organizador, gestor, dirigente...) en el deporte o en la historia de un club o asociación deportiva, modalidad deportiva o evento deportivo dentro de un pueblo, ciudad o comarca. Los documentales tenían que contar con el visto bueno del profesor.

Los grupos (de entre 4 y 6 estudiantes) los conformaron libremente los estudiantes durante la primera semana de clase. Cada grupo tenía que elaborar por escrito una propuesta de documental. En 2-3 hojas se debía indicar:

- El título del documental.

- Una breve descripción de la temática y en qué consistirá el mismo.

- La justificación de su valor, importancia y aportación.

- Señalar las fuentes de información que se tenía previsto utilizar.

- Cronograma de actividades a realizar.

- Funciones que asumiría cada miembro del equipo (director, guionista, cámara, sonido y música, edición y montaje, documentación, infografías, traducciones, subtítulos, presentador o voz en off ...).

Estas propuestas, además, fueron expuestas oralmente durante la tercera semana lectiva de la asignatura por cada equipo en una clase práctica, donde fueron analizadas por el profesor y el resto de equipos. Cada grupo obtuvo diferentes orientaciones para la elaboración de sus documentales. Algunas de estas propuestas iniciales fueron rechazadas y tuvieron que ser replanteadas y elaboradas nuevamente. 
Los estudiantes dispusieron de un mes y medio para el montaje del documental. Durante este periodo los equipos recurrieron a consultas puntuales al profesor a través del correo electrónico o alguna tutoría. Los estudiantes informaron a las personas entrevistadas sobre las características y el carácter público de los documentales. Finalizado este periodo, los estudiantes colgaron los mismos en la plataforma You Tube y el profesor los insertó en distintos posts (uno por documental) en el edublog de la asignatura para ser comentados por el resto del alumnado.

El edublog de la asignatura también contenía una pestaña con el título "Trabajo en grupo" donde se explicaba todo lo referido a la elaboración del documental y donde se enlazaban seis ejemplos de documentales relacionados con los contenidos de la asignatura. En él se indicaron los siguientes criterios de evaluación:

- originalidad y contribución al conocimiento histórico-deportivo,

- aportación de evidencias,

- contraste de las fuentes, rigor informativo y documental,

- contenidos y guión,

- calidad audiovisual y montaje,

- y cumplir con los plazos y fechas establecidas.

Como se señaló anteriormente, la participación en el edublog de la asignatura fue otro aspecto importante en la evaluación del aprendizaje del alumnado. Cada alumno o alumna tenía que realizar un total de 12 comentarios donde se valoraba el escribir con corrección ortográfica y gramatical. No se debía "cortar y pegar" de los apuntes, ni repetir información ya escrita por otros estudiantes, sino que había que debatirla, ampliarla, matizarla, corregirla, etc., desde un punto de vista personal del estudiante. En los comentarios se tenía que dar una opinión personal, pero fundamentada en las referencias bibliográficas de los temas de la asignatura. Se valoraba, especialmente, la contribución al aprendizaje de los compañeros.

\section{Resultados}

Se elaboraron un total de 9 documentales, cuyos títulos y enlace a los mismos se pueden encontrar en la tabla 1. En la experiencia participaron 48 de los 49 estudiantes matriculados en la asignatura (el 97,96\%). Es decir, prácticamente la totalidad del alumnado.

Tabla 1. Documentales elaborados por equipo y número de comentarios en el post

\begin{tabular}{lcc}
\hline \multicolumn{1}{c}{ Documentales elaborados } & $\begin{array}{c}\mathbf{N}^{\mathbf{0}} \\
\text { estudiantes } \\
\text { del equipo }\end{array}$ & $\begin{array}{c}\mathbf{N}^{\mathbf{0}} \text { de } \\
\text { comentarios } \\
\text { en el post }\end{array}$ \\
\hline $\begin{array}{l}\text { El polideportivo Nazaret y el proyecto DxE } \\
\text { https://www.youtube.com/watch?v=MlmMr_cB208 }\end{array}$ & 6 & 28 \\
$\begin{array}{l}\text { Historia del Patín Alcodiam Salesianos } \\
\text { https://www.youtube.com/watch?v=Fo3JfVjHdh0 }\end{array}$ & 4 & 18 \\
$\begin{array}{l}\text { Levante Masclets: una historia de superación } \\
\text { https://www.youtube.com/watch?v=bbKhkp5MECE }\end{array}$ & 6 & 23
\end{tabular}

(cc) EY-NC-ND 2016, Universitat Politècnica de València

Congreso In-Red (2016) 
Aprendizaje colaborativo a través de la elaboración

de documentales audiovisuales y el uso del edublog

\begin{tabular}{|c|c|c|}
\hline $\begin{array}{l}\text { Historia del Club Atletisme Xàtiva } \\
\text { https://www.youtube.com/watch?v=I7s3I8aJGyo }\end{array}$ & 6 & 17 \\
\hline $\begin{array}{l}\text { La Volta a la Marina: passió pel ciclismo } \\
\text { https://www.youtube.com/watch?v=hvCNH-bluTo }\end{array}$ & 6 & 23 \\
\hline $\begin{array}{l}\text { Història del Club Atletisme Vall d'Albaida } \\
\text { (no disponible el enlace) }\end{array}$ & 5 & 20 \\
\hline $\begin{array}{l}\text { Historia y explicación básica del tricking } \\
\text { https://www.youtube.com/watch?v=n3q_1P1S27I }\end{array}$ & 5 & 33 \\
\hline $\begin{array}{l}\text { La grada } \\
\text { https://www.youtube.com/watch?v=27CdDxJny6k }\end{array}$ & 5 & 31 \\
\hline $\begin{array}{l}\text { La colombicultura } \\
\text { https://www.youtube.com/watch?v=7GFZ3zO7Y8s }\end{array}$ & 5 & 40 \\
\hline
\end{tabular}

Los vídeos fueron subidos a la plataforma You Tube por cada uno de los equipos y, posteriormente, el profesor los insertó en posts individuales (figura 1) en el edublog de la asignatura (http://thesport2015.blogspot.com.es ${ }^{1}$ ). Con la intención de condicionar el proceso de evaluación continua, los posts del edublog estaban abiertos a los comentarios del alumnado poco más de dos semanas. Los estudiantes podían utilizar su nombre o mantener su anonimato en público a través de un pseudónimo o heterónimo (en estos casos debían informar al profesor para que pudiese identificarles para la evaluación).

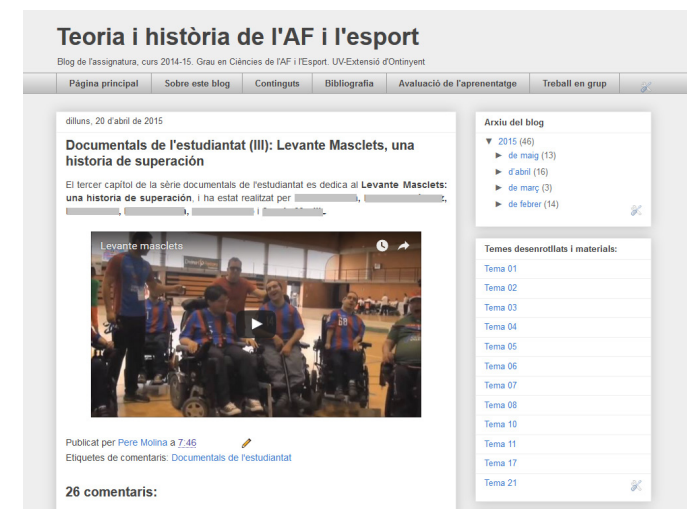

Fig. 1 Ejemplo de post de uno de los documentales en el edublog de la asignatura

En el edublog se publicaron un total de 46 posts y 662 comentarios por el alumnado; lo que se corresponde con una media de 14,39 comentarios por post y 13,79 comentarios por estudiante participante en la experiencia. La media de comentarios de estudiantes por post de los documentales se sitúa en 25,89. Es decir, los alumnos han participado más en estos posts que en los del resto del edublog. En los comentarios predominaba un tono general positivo y de felicitación a los autores de los documentales. Las críticas y los aspectos negativos tenía un carácter puntual. Este fragmento de uno de ellos puede servir de ejemplo:

\footnotetext{
1 El edublog se da de alta al inicio del curso y se cierra al finalizar el mismo. El edublog del curso 2014-15 ha dejado de ser accesible públicamente. Se puede solicitar el acceso para su consulta a través de la dirección juan.p.molina@uv.es
}

(cc) EY-NC-ND 2016, Universitat Politècnica de València 
Este documental lo resumiría en una palabra: EMOTIVO. La verdad es que ha sido un documental que me ha emocionado, pienso que las personas con discapacidad deberían tener muchas más oportunidades y recursos para realizar deporte. (...)

Me gusta que RI y su equipo sean competitivos, que se cabreen cuando pierden, pero que también sean muy deportivos, que sepan aprovechar la derrota para mejorar mucho más.

Destacar la actuación del presentador SM, que tiene una gran facilidad para expresarse y hacer que las personas que veamos el documental tengamos empatía por estas personas.

También me gusta el montaje del video, pero pienso que hay demasiadas entrevistas. Por último quisiera dar las gracias a mis compañeros por dar a conocer el deporte para discapacitados y al Levante UD por dar la oportunidad a estas personas de disfrutar del deporte. (AOS)

Otro hallazgo de la presente experiencia y que no estaba previsto de antemano, tuvo que ver con la evaluación. Como sabemos, la evaluación es un aspecto muy ligado a la metodología de enseñanza. El aprendizaje colaborativo requiere de una forma de evaluación acorde con sus características y necesidades. En principio, se tenía previsto que la evaluación de los documentales la realizase el profesor, de tal forma que la nota fuese establecida al conjunto del grupo, valorándose el documental de 0 a 3 puntos. Para atender a una evaluación colaborativa, siempre que la calificación asignada por el profesor superase el 1,5 (de esos 30 puntos totales), el equipo de estudiantes tenía la opción de reajustar la calificación entre sus miembros, previo acuerdo entre ellos y siempre que el resultado de la media de calificaciones del grupo se correspondiese con la calificación otorgada por el profesor al equipo y no quedase ningún estudiante con menos de 1,5 puntos.

Sin embargo, dada la implicación de los estudiantes en esta experiencia, tanto en la elaboración de los documentales como en los comentarios posteriores, y dada la calidad de los documentales presentados, el profesor propuso al alumnado ampliar su participación en el proceso de evaluación. El alumnado aceptó y se realizó una coevaluación entre estudiantes. El profesor facilitó unas planillas donde cada equipo tenía que valorar el resto de documentales entre 0 y 3 puntos. Las valoraciones de cada grupo eran confidenciales. El profesor también realizó la valoración de cada uno de los documentales, siendo una calificación más, con el mismo peso que la de cualquier equipo de estudiantes. La calificación final de cada documental fue la nota media de las ocho de los equipos restantes y la del profesor.

Como puede observarse en la tabla 2, la calificación media otorgada por el profesor es la segunda más alta de las 10. En ella también se puede observar que los grupos que mejor valoran el trabajo de sus compañeros son los que obtienen calificaciones más bajas en sus documentales.

Tabla 2. Valoración media otorgada por equipo y la calificación obtenida en su documental

\begin{tabular}{ccc}
\hline Equipo & $\begin{array}{c}\text { Valoración media otorgada } \\
\text { al resto de equipos }\end{array}$ & Calificación obtenida \\
\hline A & 2,06 & 2,65 \\
B & 2,28 & 2,52 \\
C & 2,08 & 2,41 \\
D & 1,98 & 2,30 \\
E & 2,08 & 2,26
\end{tabular}

(cc) EY-NC-ND 2016, Universitat Politècnica de València

Congreso In-Red (2016) 


\begin{tabular}{ccc} 
F & 2,03 & 2,21 \\
G & 2,29 & 1,96 \\
H & 2,34 & 1,93 \\
I & 2,46 & 1,81 \\
Profesor & 2,42 & - \\
\hline
\end{tabular}

También se observa que los estudiantes resultaron más exigentes que el profesor en la valoración. Prueba de ello fue que sólo un grupo otorgó la máxima puntuación a otro y tres equipos asignaron 2,8 puntos al trabajo de otros compañeros. Por el contrario, la nota más baja asignada a un equipo por otro fue de un 1 . La segunda nota más baja fue un 1,5 que fue concedida por cuatro equipos. Por su parte, el profesor otorgó un 3 a un documental y un 2,75 a tres. La calificación más baja del profesor fue un 1,75 a uno de los documentales.

\section{Conclusiones}

Como conclusiones de la experiencia descrita destacamos:

1) La alta participación del alumnado en la experiencia propuesta $(97,96 \%)$, la gran implicación en la elaboración de los documentales y también en la realización de comentarios individuales a los mismos en posts específicos publicados en el edublog de la asignatura, cuya media de comentarios a estos posts $(25,89)$ fue más alta que la media general de comentarios a los posts del edublog, elaborados por el profesor sobre otras cuestiones relacionadas con la asignatura $(14,39)$. Esto demuestra un mayor interés por parte del alumnado hacia contenidos desarrollados por ellos mismos que por el profesor.

2) El planteamiento de este tipo de tareas, por parte del profesor, posibilita una mayor libertad del alumnado en la elección de temáticas, que puedan ajustarse mejor a sus expectativas personales de aprendizaje de conocimientos. Asimismo, puede suponer una mejora en la relación personal del profesor con el alumnado, a partir de la búsqueda de consensos para el establecimento de las temáticas objeto de estudio.

3) Cabe destacar la buena calidad de la mayor parte de los 9 documentales que se realizaron, a juzgar por la valoración del profesor de la asignatura, y también por el contenido de los comentarios publicados en los posts y por la coevaluación que realizaron los diferentes equipos sobre los documentales de sus compañeros.

4) El planteamiento de una evaluación colaborativa y compartida entre el alumnado y el profesor en procesos de enseñanza-aprendizaje, se ha traducido, en nuestro caso, en un mayor sentido de la responsabilidad y de justicia sobre el trabajo personal de los estudiantes y del trabajo de los compañeros. 


\section{Referencias}

ANTOLÍN, L., MOLINA, P., VILlAMÓN, M., DEVÍS, J. y PÉREZ-SAMANIEGO, V. (2011). "Uso de blogs en ciencias de la actividad física y el deporte". @tic. Revista d'innovació educativa, 7, $12-18$.

BERMÚDEZ, N. (2010). "El documental histórico: una propuesta para la reconstrucción audiovisual de la historia petrolera de Zulia". Omnia, 16(2), 113-131.

DEL VILLAR, F. (Coord.) (2007). Libro blanco Título de Grado en Ciencias de la Actividad Física y del Deporte. Madrid: ANECA.

DILLENBOURG, P. (1999). "What do you mean by collaborative learning?". En P. Dillenbourg (Ed.) Collaborative-learning: Cognitive and Computational Approaches. Oxford: Elsevier.

JOHNSON, D. W., JOHNSON, R. T. y HOLUBEC E. J. (1998). Advanced cooperative learning ( $2^{\mathrm{a}}$ ed). Edina, MN: Interaction Book Company.

KAGAN, S. (1989). "The structural approach to cooperative learning". Educational leadership, 47(4), 12-15.

LAAL, M. y LAAL, M. (2012). "Collaborative learning: what is it?". Journal of Procedia-Social and Behavioral Sciences, 31, 491-495

MARTINEZ-BAENA, A. y MOLINA, P. (2015). "El uso del edublog y el aprendizaje colaborativo en clases prácticas de Educación Superior". XXIII Jornadas Universitarias de Tecnología Educativa. Badajoz, 11 y 12 de junio. Disponible en: <http://eventos.unex.es/file_manager/get paper/2211> [Consulta: 30 de marzo de 2016].

MOlinA, P., ANTOlin, L., PEREZ-SAMANIEGO, V., DEVIS, J., VILlAMON, M. у VALENCIANO, J. (2013). "Uso de blogs y evaluación continua del aprendizaje del alumnado universitario". EDUTEC, Revista Electrónica de Tecnología Educativa, 43.

MOLINA, J. P., VALENCIA-PERIS, A. y SUAREZ, C. (2016). "Percepción de los estudiantes de una experiencia de uso didáctico de blog docente en Educación Superior”. Educación XX1, 19(1), 91113.

MOLINA, P., VALENCIA-PERIS, A, VALENCIANO, J., PEREZ-SAMANIEGO, V. y DEVIS, J. (2012). "Evolución de una experiencia universitaria de innovación educativa con blogs". En D. Cobos, E. López, A. Jaén, A. Hilario, M. Padilla y L. Molina (dir.), Actas del I Congreso Internacional Virtual sobre innovación pedagógica y praxis educativa Innovagogía 2012 (pp. 745756). Sevilla: AFOE. < https://drive.google.com/file/d/0Bw2Ce9zDtnSTbWtlZldwanQwNIU/view> [Consulta: 30 de marzo de 2016]

MOLINA, P., VALENCIANO, J. y VALENCIA-PERIS, A. (2015). "Los blogs como entornos virtuales de enseñanza y aprendizaje en Educación Superior”. Revista Complutense de Educación, 26, 15-31.

SLAVIN, R. (1990). Cooperative learning: Theory, research and practice. Englewood Cliffs, NJ: Prentice Hall.

SMITH, B. L. y MACGREGOR, J. T. (1992). “What is Collaborative Learning?”. En A. Goodsell, M. Mahler, V. Tinto, B. L. Smith y J. MacGregor (Eds). Collaborative Learning: A Sourcebook for

(cc) BY-NC-ND 2016, Universitat Politècnica de València

Congreso In-Red (2016) 
Aprendizaje colaborativo a través de la elaboración

de documentales audiovisuales y el uso del edublog

Higher Education (pp. 9-22). Pennsylvania State University, USA: National Center on Postsecondary Teaching, Learning, and Assessment Publishing.

SUAREZ, C. y GROS, B. (2013). Aprender en red. De la interacción a la colaboración. Barcelona: Universitat Oberta de Catalunya.

(cc) EY-NC-ND 2016, Universitat Politècnica de València 